Supplement of Atmos. Meas. Tech. Discuss., 8, 5735-5768, 2015

http://www.atmos-meas-tech-discuss.net/8/5735/2015/

doi:10.5194/amtd-8-5735-2015-supplement

(C) Author(s) 2015. CC Attribution 3.0 License.

(c) (i)

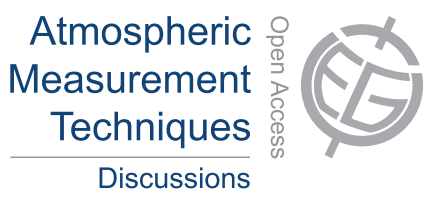

Supplement of

\title{
Characterization of trace metals with the SP-AMS: detection and quantification
}

\author{
S. Carbone et al.
}

Correspondence to: S. Carbone (carbone@if.usp.br)

The copyright of individual parts of the supplement might differ from the CC-BY 3.0 licence. 


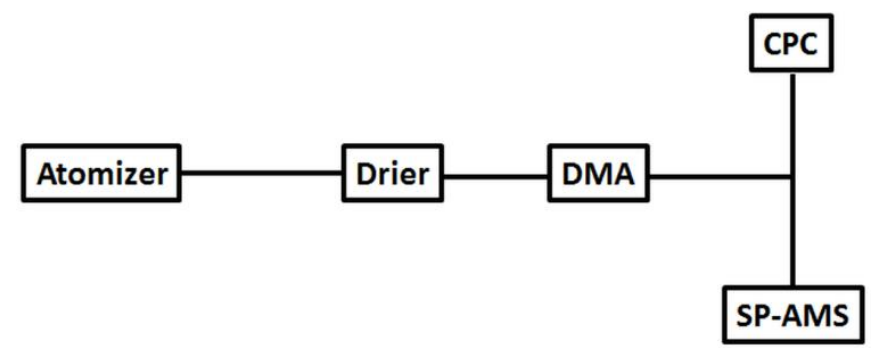

Figure S1 - Instrument setup to measure trace elements in the laboratory.

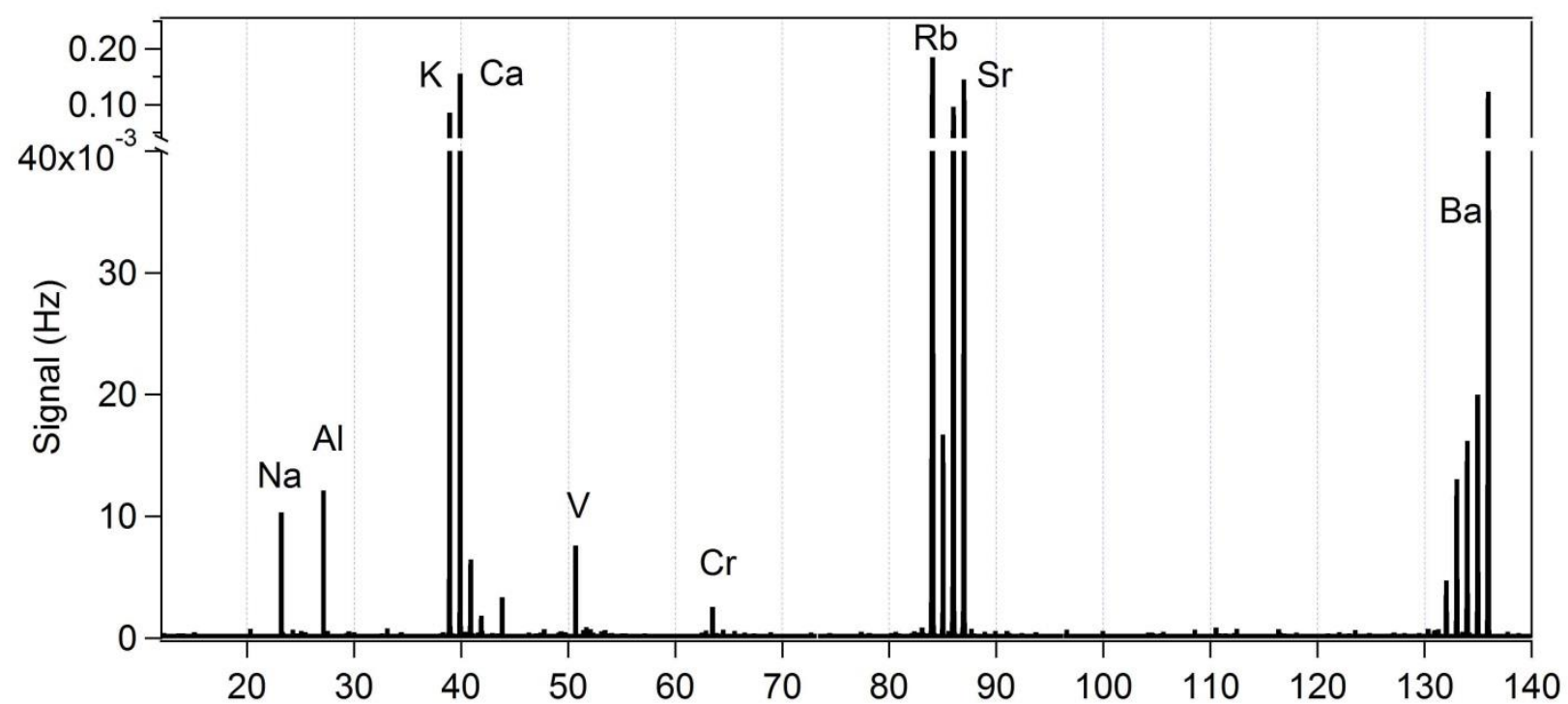

Figure S2 - Mass spectrum obtained with Tungsten and filament switched off and laser vaporizer on. 


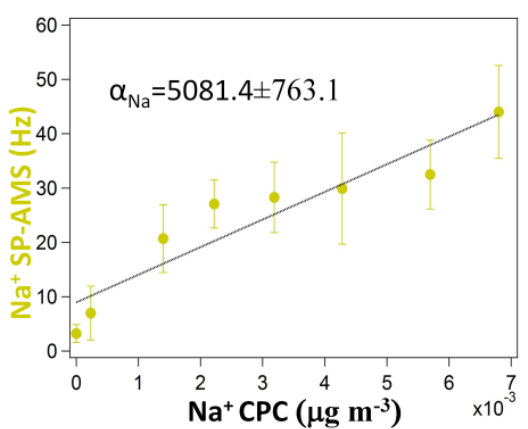

(a)

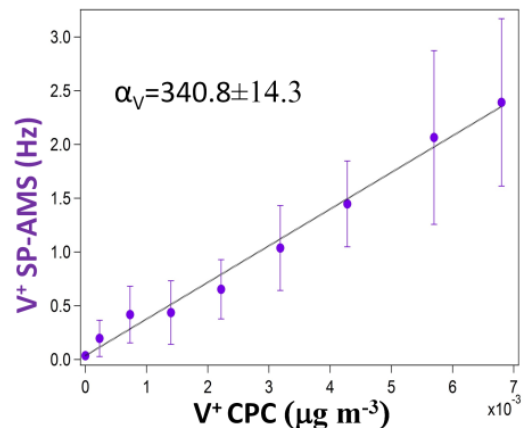

(d)

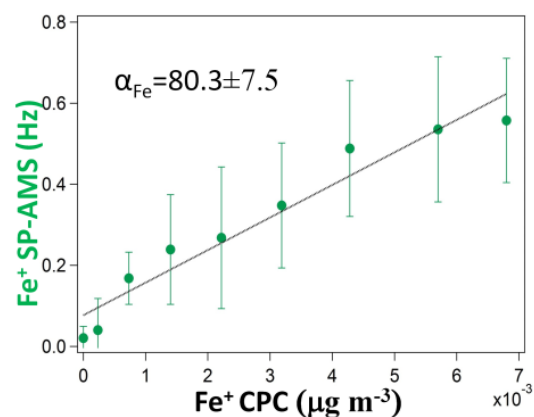

(g)

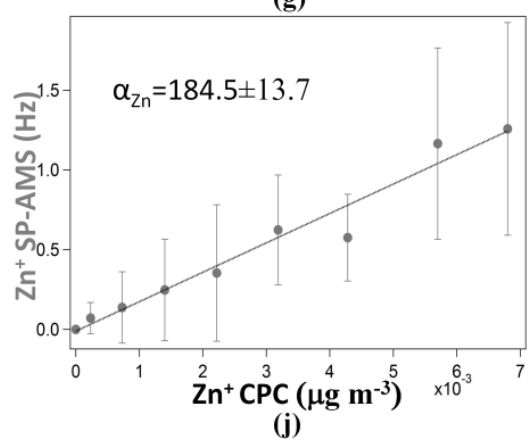

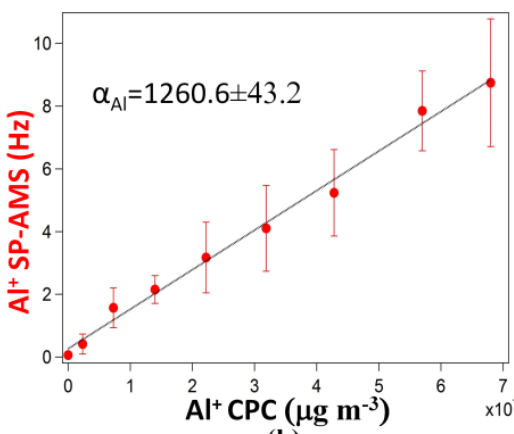

(b)

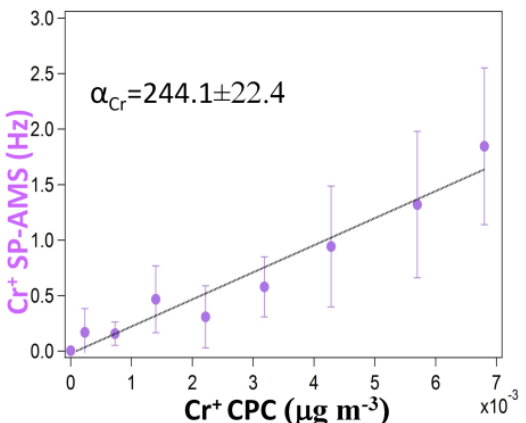

(e)

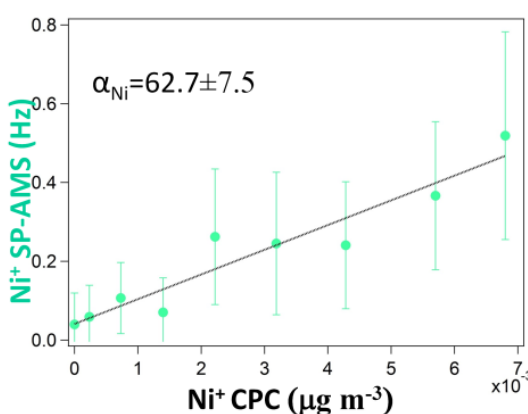

(h)

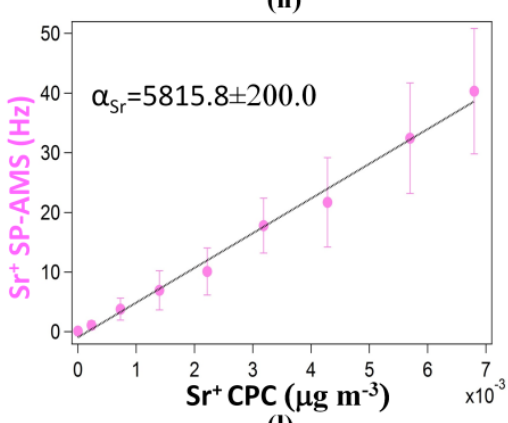

(l)

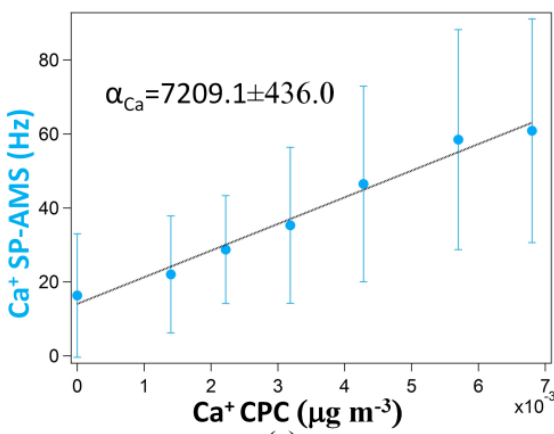

(c)

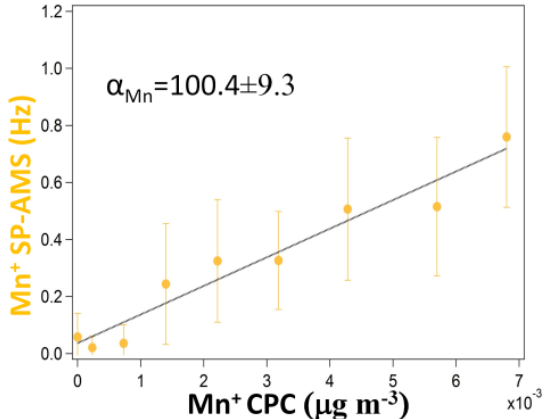

(f)

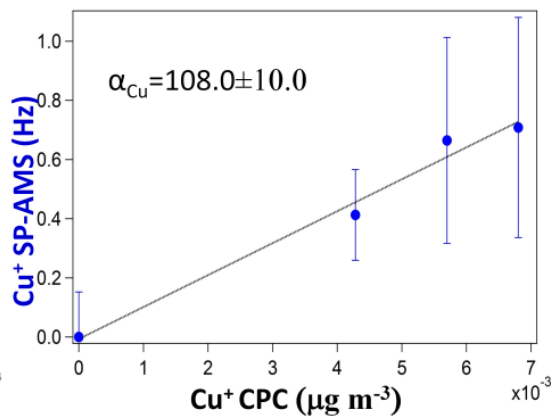

(i)

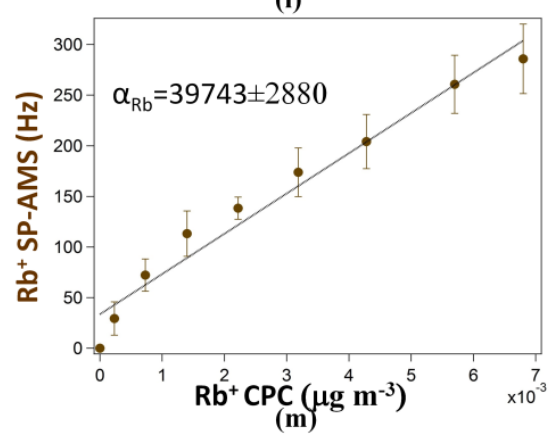

Figure S3- Signal measured by the SP-AMS $(\mathrm{Hz})$ versus the mass concentration obtained by the CPC $\left(\mu \mathrm{g} \mathrm{m}^{-3}\right)$ for each trace element and respective slope values $(\alpha)$. 


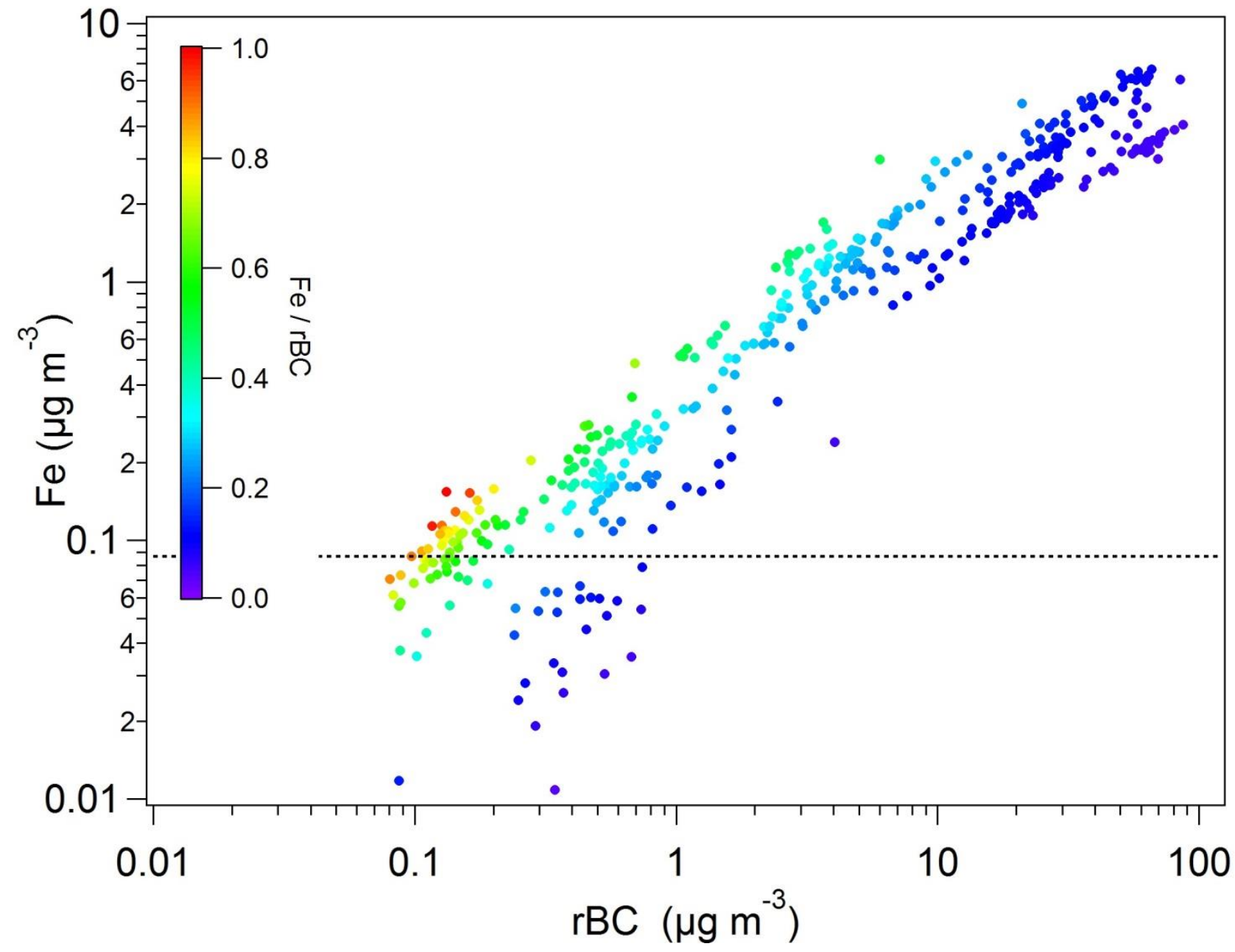

Figure $\mathrm{S} 4-\mathrm{Fe}$ mass concentration as a function or $\mathrm{rBC}$ concentration measured at the heating station with the SP-AMS. The dashed black line represent the Fe limit of detection for this instrument. 
Table S1- Relative atomic mass, isotopic composition, standard concentration, nitric acid concentration and identified isotopes of the trace elements investigated in this study.

\begin{tabular}{|c|c|c|c|c|c|}
\hline Element & $\begin{array}{l}\text { Relative } \\
\text { atomic } \\
\text { mass }\end{array}$ & $\begin{array}{c}\text { Isotopic } \\
\text { composition } \\
(\%)^{a}\end{array}$ & 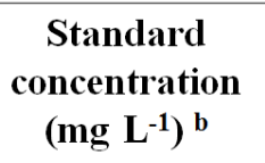 & $\begin{array}{c}\mathrm{HNO}_{3} \\
\text { concentration } \\
(\%, \mathbf{w} / \mathbf{w})^{b}\end{array}$ & $\begin{array}{c}\text { Identified isotopes with } \\
\text { the SP-AMS }\end{array}$ \\
\hline $\mathrm{Na}$ & 22.9897 & 100.00 & 1000 & 2 & ${ }^{23} \mathrm{Na}^{+}$ \\
\hline Al & 26.9815 & 100.00 & 1000 & 2 & ${ }^{27} \mathrm{Al}^{+}$ \\
\hline Ca & 39.9625 & 96.94 & 1000 & 2 & ${ }^{40} \mathrm{Ca}^{+},{ }^{42} \mathrm{Ca}^{+},{ }^{44} \mathrm{Ca}^{+}$ \\
\hline $\mathbf{V}$ & 50.9439 & 99.75 & 1000 & 3 & ${ }^{51} \mathrm{~V}^{+}$ \\
\hline $\mathrm{Cr}$ & 51.9405 & 83.78 & 1000 & 2 & ${ }^{52} \mathrm{Cr}^{+},{ }^{53} \mathrm{Cr}^{+},{ }^{54} \mathrm{Cr}^{+}$ \\
\hline Mn & 54.9380 & 100.00 & 998 & 2 & ${ }^{55} \mathrm{Mn}^{+}$ \\
\hline $\mathrm{Fe}$ & 55.9349 & 91.75 & 1000 & 2 & ${ }^{54} \mathrm{Fe}^{+},{ }^{55} \mathrm{Fe}^{+},{ }^{56} \mathrm{Fe}^{+},{ }^{57} \mathrm{Fe}^{+}$, \\
\hline $\mathbf{N i}$ & 57.9353 & 68.07 & 1000 & 2 & $\begin{array}{l}{ }^{58} \mathrm{Ni}^{+},{ }^{60} \mathrm{Ni}^{+},{ }^{61} \mathrm{Ni}^{+}, \\
{ }^{62} \mathrm{Ni}^{+},{ }^{64} \mathrm{Ni}^{+}\end{array}$ \\
\hline $\mathbf{C u}$ & 62.9295 & 69.15 & 1000 & 2 & ${ }^{63} \mathrm{Cu}^{+},{ }^{64} \mathrm{Cu}^{+}$ \\
\hline Zn & 63.9291 & 48.26 & 1000 & 2 & $\begin{array}{l}{ }^{64} \mathrm{Zn}^{+},{ }^{66} \mathrm{Zn}^{+},{ }^{67} \mathrm{Zn}^{+},{ }^{68} \mathrm{Zn}^{+}, \\
{ }^{70} \mathrm{Zn}^{+}\end{array}$ \\
\hline $\mathbf{R b}$ & 84.9117 & 72.17 & 1000 & 2 & ${ }^{85} \mathrm{Rb}^{+},{ }^{87} \mathrm{Rb}^{+}$ \\
\hline $\mathrm{Sr}$ & 87.9056 & 82.58 & 1000 & 2 & ${ }^{84} \mathrm{Sr}^{+},{ }^{86} \mathrm{Sr}^{+},{ }^{87} \mathrm{Sr}^{+},{ }^{88} \mathrm{Sr}^{+}$ \\
\hline Ba & 137.9052 & 71.69 & 1000 & 2 & $\begin{array}{l}{ }^{130} \mathrm{Ba}^{+},{ }^{132} \mathrm{Ba}^{+},{ }^{134} \mathrm{Ba}^{+}, \\
{ }^{135} \mathrm{Ba}^{+},{ }^{136} \mathrm{Ba}^{+},{ }^{137} \mathrm{Ba}^{+}, \\
{ }^{138} \mathrm{Ba}^{+}\end{array}$ \\
\hline
\end{tabular}

${ }^{a}$ Watson et al., 2004.

${ }^{\mathrm{b}}$ Sigma-Aldrich standards in nitric acid. 